Artículo de Revisión

Apuntes del CENES

ISSN 0120-3053

Volumen $33-\mathrm{N}^{\circ} .57$

Enero - Junio de 2014

Págs. 117-146

\title{
¿Han sido los mercados bursátiles eficientes informacionalmente?
}

\author{
Have been informationally \\ efficient stock markets?
}

Juan Benjamín Duarte Duarte * Juan Manuel Mascareñas Pérez Iñigo **

Fecha de recepción: 30 de septiembre de 2013

Concepto de evaluación: 18 de febrero de 2014

Fecha de aprobación: 21 de marzo de 2014

\footnotetext{
Doctor en Finanzas de Empresas. Docente de Planta. Universidad Industrial de Santander. Ciudad Universitaria, Edificio Ingeniería. Bucaramanga, Colombia. Correo electrónico: jbduarted@hotmail.com

** Doctor en Ciencias Económicas y Empresariales. Catedrático de Economía Financiera. Universidad Complutense de Madrid. Facultad de Ciencias Económicas. Universidad Complutense. Madrid. España. Correo electrónico: jmascare@ucm.es
} 


\section{Resumen}

En el presente trabajo se estudia la contrastación de la eficiencia de mercados bursátiles en los últimos quince años, para ello se acude a la revisión de artículos de la base de datos ScienceDirect caracterizando los resultados de forma porcentual. Se encuentra que el $60 \%$ de los trabajos rechaza la eficiencia del mercado, el $35 \%$ presenta evidencia de eficiencia, y el $5 \%$ restante verifica una mejora progresiva de la eficiencia debida a reformas económicas, mayor velocidad en el flujo de información y el lanzamiento de nuevos productos financieros.

Palabras clave: hipótesis del mercado eficiente, mercados financieros.

JEL: D52, G14, G15.

\section{Abstract}

In this paper we study the testing of the stock market efficiency in the last fifteen years, for that we review the papers of ScienceDirect database to characterize the results in percent. We find that $60 \%$ of papers rejects stock market efficiency, $35 \%$ presents evidence of efficiency, and the remaining $5 \%$ verifies a progressive improvement in efficiency due to economic reforms, faster information flow and the launch of new financial products.

Keywords: efficient market hypothesis, financial markets. 


\section{INTRODUCCIÓN}

Los modelos de valoración de activos como el CAPM y el APT parten del supuesto de que los mercados son eficientes informacionalmente, principio que ha evolucionado en los últimos cien años desde que Bachelier (1900) desarrollara en su tesis doctoral la fundamentación matemática y estadística del movimiento browniano, explicando la eficiencia de los mercados en función del comportamiento de una martingala. Años más tarde, Cowles (1933) estudia empíricamente el nivel de asertividad de las recomendaciones de los analistas de bolsa, y concluye que sus opiniones no pronosticaban sistemáticamente el mercado, reforzando así, para ese entonces, la teoría de que el mercado norteamericano era eficiente. Posteriormente, Fama (1965) define un mercado eficiente como una competencia equitativa, en la cual la información está libremente disponible para todos los participantes, los cuales intentan predecir los valores futuros de los activos del mercado haciendo que en un instante del tiempo, los precios actuales reflejen los efectos de toda la información disponible basada en eventos ocurridos y eventos que se esperan tendrán lugar en el futuro. En otras palabras, en un momento dado el precio actual del activo será un buen estimador de su valor intrínseco. Desde Fama, han sido innumerables los trabajos llevados a cabo sobre la comprobación de la eficiencia de los mercados bursátiles, con el principal objetivo de brindar un elemento de juicio para que inversionistas e instituciones reguladoras de la bolsa evalúen si se están dando o no las condiciones ideales en cuanto a lo que se denomina "juego justo". Por tal motivo, el propósito de este trabajo es revisar las investigaciones empíricas más 
relevantes que se han llevado a cabo hasta el año 2012 a nivel de mercados individuales $y$ por regiones (Norteamérica, Europa, Asia, etc.), buscando detectar cuáles han sido los hallazgos más significativos.

El trabajo está organizado en cuatro capítulos, en el primero se presenta la introducción, el segundo capítulo expone la teoría de mercados eficientes, el tercero se ocupa de hacer una revisión de la literatura que se ha publicado en los últimos quince años, sobre la contrastación de la hipótesis de eficiencia en los mercados bursátiles en países y regiones tanto desarrolladas como no desarrolladas, y por último se exponen los hallazgos y conclusiones más importantes del estudio.

\section{LA TEORÍA DE LOS MERCADOS EFICIENTES}

La definición de mercado eficiente está asociada al concepto de igualdad de condiciones postulado por Cardano (1953) como un principio fundamental de los juegos de azar. Trasladando este principio a los mercados bursátiles, podría decirse que es la idea subyacente de la teoría de mercados bursátiles eficientes, dado que la igualdad de condiciones de tipo informacional hace que el mercado de valores sea "un juego justo", en el cual ningún agente puede obtener sistemáticamente ganancias extraordinarias a partir de cualquier tipo de información. La definición moderna de mercado eficiente está fundamentada en los aportes de autores como Bachelier (1900), Samuelson (1965), Mandelbrot (1963), Malkiel (1992) y Fama (1965, 1991); quienes en general plantean que un mercado es eficiente cuando en él se cuenta con la suficiente liquidez y racionalidad económica por parte de los agentes como para que cualquier tipo de información relevante sea absorbida por los precios de forma instantánea, y genere un comportamiento aleatorio en ellos, lo que hace imposible su pronóstico sistemático.

\section{Clasificación de la eficiencia del mercado}

La eficiencia de mercado ha sido estructurada según el grado de información con que se cuente en la formación de los precios. Es así como Roberts $(1967)^{1}$ plantea la distinción entre eficiencia fuerte y débil, sin embargo Fama (1970) retoma y amplía la definición a los tres niveles de eficiencia de acuerdo con el concepto fully reflect: la forma débil (weak form) plantea que la información relevante para tomar decisiones de inversión está recogida en los precios históricos de las acciones, mientras que la forma semifuerte (semistrong) se presenta cuando, además de los precios históricos, se cuenta con información pública que contribuye a la

Fama (1970) reconoce que el primero en hacer la distinción de los tipos de eficiencia fue Roberts (1967). 
construcción de los precios. Por último, la forma fuerte (strong) de eficiencia del mercado se define como aquella en que los inversores cuentan con información privilegiada relevante para la estimación de los precios fundamentales. Es decir, la forma fuerte contiene a la semifuerte y esta última implica el cumplimiento de la forma débil.

Supuestos de la hipótesis de mercado eficiente

La hipótesis de mercado eficiente se fundamenta en varios supuestos, que son planteados principalmente por Ludlow (1997) y Fama (1970):

Desde el punto de vista institucional:

1. Los precios son libres, se forman por oferta y demanda sin ningún tipo de intervención o restricción tales como las bandas de precios analizadas por Chen, Gerlach, y Liu (2011) en algunos mercados asiáticos.

2. Hay libre entrada y salida del mercado, sin limitación a los agentes para comprar o vender los activos en cualquier momento.

3. El mercado provee toda la información relevante sin coste, las instituciones que administran e intervienen en los mercados procuran que la información relevante de cada activo esté disponible para el conocimiento de los agentes de forma "gratuita". En este sentido el internet ha prestado un gran apoyo para la divulgación de la gestión empresarial a través de las páginas web de cada compañía.

Desde el punto de vista de los agentes:

1. Los inversionistas hacen uso de toda la información relevante, sin embargo se debe tener en cuenta que un inversionista considera la información importante dependiendo de su perfil (Peters, 1994).

2. El uso que se hace de la información es económicamente correcto, es decir, los inversionistas son racionales y están de acuerdo en los principios de análisis de la información económica para llegar a precios cercanos al fundamental.

\section{Cuestionamientos a la hipótesis del mercado eficiente}

Los premios nobel de economía del 2001, Akerlof (1970), Spence (1973), Stiglitz y Rothschild (1976), cuestionan la hipótesis de mercado eficiente, detectando asimetrías en la información que generan problemas de riesgo moral y de selección adversa en los mercados de automóviles, laboral y de crédito, respectivamente. Además, Grossman y Stiglitz (1980) critican la hipótesis de eficiencia principalmente en su forma fuerte, argumentando que los precios reflejan parcialmente la información disponible, cuestionamiento que es presentado como una paradoja por Uribe y Ulloa (2011): 
Si se parte del supuesto de que la información es costosa y los mercados son eficientes, entonces nadie tendrá incentivos para incurrir en el coste de la información. Será más rentable $\mathrm{y}$, por ende, un comportamiento racional, esperar a que los precios reflejen toda la información disponible, incluida la información que es costosa. No habrá que esperar mucho en todo caso, porque la EMH garantiza que esta información se incorpora en el precio instantáneamente. No obstante, puesto que todos los agentes tienen los mismos incentivos, ninguno incurrirá en el coste de la información, razón por la cual será imposible que los precios la reflejen.

Considerando que Grossman y Stiglitz parten de que la información es costosa, este cuestionamiento no iría en contra de la eficiencia débil y semifuerte, para las cuales la información generalmente se publica sin coste, tal como lo reconoce Fama (1991).

Por otro lado, se ha encontrado evidencia de que en varios mercados los agentes no son racionales en el sentido económico, sino que toman decisiones basados en elementos psicológicos como los efectos manada o moda, tal como lo comprueban Kaminsky y Schmukler (1999), negociando por entusiasmo o imitación e induciendo a que los precios suban o bajen a niveles alejados de su valor intrínseco; adicionalmente Stiglitz
(2010) desmiente teorías racionales del mercado (incluyendo la EMH) basándose en el comportamiento imprudente y descontrolado de Wall Street en los años previos al 2007, que originaron la crisis de las subprimes en Estados Unidos y que según Stiglitz, fue adicionalmente mal manejada por los respectivos gobiernos, ya que para salir de la crisis se castigaron los salarios y se redujeron las pensiones, mientras que se incrementaron los bonos de los banqueros. No obstante en contra de estas detracciones, Shleifer (2003) aclara que efectivamente existen inversionistas no racionales que generan operaciones al azar, pero que éstas son anuladas tanto por inversionistas racionales como por arbitrajistas (quienes son mayoría en el mercado), y por lo tanto su influencia sobre los precios es limitada, tal como explican Aragonés y Mascareñas (1994), quienes afirman que las acciones de los inversores inteligentes son las que impulsan a los precios del mercado a aproximarse a sus valores intrínsecos.

Otro cuestionamiento a la teoría de mercados eficientes está dirigido a que los inversionistas principales delegan casi todas las decisiones sobre sus negociaciones a intermediarios profesionales (corredores de bolsa, bancos, administradores de fondos), generando un "problema de teoría de agencia", en donde los intereses de los intermediarios no siempre son iguales a los de los inversionistas; ante esta disyuntiva se sugiere que los modelos de fijación de precios de activos tengan en 
cuenta a los intermediarios, tal como lo proponen Vayanos y Woolley (2013).

Algunas de las anteriores críticas, como la expuesta por Stiglitz, ponen de manifiesto que la regulación del estado en los mercados financieros podría contribuir a un mayor nivel de eficiencia, sin embargo desde el punto de vista de los agentes, una fuerte regulación podría generar ineficiencias en estos mercados, tal como lo han comprobado Chong, Lam y Yan (2012) en China.

\section{ESTUDIOS EMPÍRICOS DE LA} TEORÍA DE MERCADO EFICIENTE

En esta sección se revisan los estudios empíricos acerca de la hipótesis de mercado eficiente desde 1933 hasta el año 2012.

\section{Primeros estudios}

Cowles (1933) fue uno de los primeros en estudiar la eficiencia de los mercados de forma empírica al evaluar el nivel de asertividad de los analistas de bolsa de su tiempo, concluyendo que sus recomendaciones no predecían sistemáticamente el mercado, reforzando así la teoría de que el mercado americano era eficiente para esa época. Posteriormente, Kendall (1953), analiza 22 compañías norteamericanas, encontrando aleatoriedad y estacionalidad $^{2}$ en las series de precios. Años más tarde Roberts (1959) ratifica lo hallado por Kendall, al demostrar que las series financieras son semejantes al recorrido aleatorio. Igualmente Osborne (1959) encuentra que el comportamiento de los precios de los activos es similar al movimiento browniano.

En los años sesenta Mandelbrot (1963) y Samuelson (1965) asociaron modelos matemáticos como la martingala al comportamiento de las series financieras, mientras que Fama $(1965,1970)$ compiló en sus publicaciones los fundamentos empíricos y teóricos desarrollados hasta la fecha, proponiendo el modelo de recorrido aleatorio como herramienta para describir el comportamiento de los mercados bursátiles.

\section{COMPROBACIÓN DE LA EFICIENCIA DEL MERCADO DESDE 1997}

A partir de la búsqueda en la base de datos ScienceDirect ${ }^{3}$, se revisan los principales estudios sobre eficiencia de mercado llevados a cabo a nivel mundial.

\section{Eficiencia de mercado por regiones}

La eficiencia de los mercados bursátiles ha sido ampliamente testeada desde su formulación en la década de los sesenta,

\footnotetext{
Se le atribuye como el primero en detectar la estacionalidad en los precios bursátiles.

3 Esta base de datos cuenta con 3178 revistas indexadas, de las cuales 344 incluyen temas de economía, econometría y finanzas.
} 
tanto en mercados desarrollados (Norteamérica y Europa), como en emergentes (Asia, Oceanía, África y Latinoamérica). En los siguientes numerales se analizan los trabajos llevados a cabo en estas regiones con el fin de detectar posibles patrones que ayuden a comprender mejor el comportamiento de los mercados desde el punto de vista de su eficiencia.

\section{Norteamérica}

La bolsa norteamericana representada a través de los índices SyP500, Dow Jones, NYSE, Nasdaq y SyP/TSX de Canadá, generalmente es tomada como referente de mercado eficiente, teniendo en cuenta su mayor liquidez y volumen de negociación a nivel mundial. No obstante, la eficiencia del mercado norteamericano ha sido contrastada continuamente con resultados contradictorios por diferentes metodologías.

Usando análisis técnico Potvin, Soriano y Vallée (2004) generan normas comerciales por programación genética para empresas canadienses del TSE 300 Index entre 1992 y 2000 evidenciando que estas reglas son generalmente beneficiosas cuando el mercado cae o cuando es estable y que además superan al enfoque de comprar y mantener. De igual forma $\mathrm{Wu}$, Huang y Chiu (2011) usando reglas de filtro con media móvil y árboles de decisión en el Nasdaq encuentran que el método de negociación propuesto genera beneficios extras. También Shynkevich (2012) combina análisis técnico con el efecto tamaño de empresa, encontrando que las carteras de acciones de empresas pequeñas son más predictivas que las compuestas por las grandes en los índices NYSE, AMEX y NASDAQ; además este autor concluye que a partir del año 2000 el mercado se hace menos previsible, mejorando su eficiencia. Estos tres estudios ratifican la no eficiencia del mercado norteamericano tal como lo evidenció Brock, Lakonishok, y LeBaron (1992) quien fue uno de los primeros en rechazar la eficiencia del mercado a partir del análisis técnico. Por el contrario, la eficiencia del mercado norteamericano no ha sido rechazada por autores como Day y Wang (2002) y Marshall, Young y Rose (2006) quienes mediante el uso normas técnicas como media móvil, Trading Range Break-Out y análisis velas, no encuentran posibilidades de obtener ganancias extraordinarias de forma sistemática.

Igualmente, otros autores valiéndose de modelos de predicción han encontrado resultados contradictorios en la eficiencia del mercado norteamericano. Por una parte, Gençay (1998) analiza el mercado Dow Jones Industrial Average Index desde 1897 a 1988, para evaluar la predictibilidad lineal y no lineal mediante técnicas de media móvil y modelos GARCH-M; así mismo Ansari, Kumar, Shukla, Dhar y Tiwari (2010) realizan pronósticos en el mercado de valores del NASDAQ entre 2006 y 2008, mediante modelos ARCH y redes neuronales; ambos estudios encuentran evidencia de 
predictibilidad no lineal. Por otra parte, Coakley y Fuertes (2006) usando modelos de predicción autorregresivos y Kim, Shamsuddin y Lim (2011) mediante ratio de varianza, matizan la eficiencia de acuerdo a la condición económica del mercado, encontrando que en periodos de crisis los precios de los activos se aproximan a su valor fundamental, mientras que en periodos alcistas se hacen más predecibles.

Otros trabajos que se concentran en el análisis de la estructura de las series financieras mediante el estudio de la memoria de largo plazo $^{4}$ y la aleatoriedad (Rachas, BDS) están divididos en cuanto a la eficiencia del mercado dado que Kohers, Pandey, y Kohers (1997) analizan las empresas del NYSE y el mercado Over-the-Counter buscando simultáneamente comportamiento caótico y efecto tamaño de empresa, sus resultados sugieren que las firmas grandes del NYSE presentan una influencia determinista por lo que serían potencialmente predecibles; así mismo Serletis y Shintani (2003) mediante el test BDS y el test Lyapunov rechazan el comportamiento caótico en las series pero identifican dependencia lineal útil para diseñar modelos de predicción estocásticos en las series del Dow Jones entre 1928 y 2000; igualmente Mulligan y Lombardo (2004) detectan una reacción exagerada en las acciones del sector marítimo de NASDAQ, NYSE y
AMEX desde 1988 a 2002 usando el análisis de Hurst. Por el contrario, autores como Couillard y Davison (2005) no rechazan la hipótesis de eficiencia débil en el SyP500 a partir del coeficiente de Hurst en el periodo de 1973 a 2003, a esta misma conclusión llega Liu (2007) quien estudia las acciones extranjeras listadas en el mercado de Estados Unidos mediante el test de rachas para el periodo de 1970 a 2005.

En cuanto a la comprobación de la eficiencia semifuerte del mercado americano (NYSE, NASDAQ y AMEX) los resultados muestran una evolución positiva de esta hipótesis. Easley, Kiefer, y O'Hara (1997) en los meses de octubre a diciembre de 1990, hallan retornos anormales ante los anuncios de eventos en el volumen y el tamaño de las negociaciones, años después, Ferreira y Brooks (1999) detectan retornos significativamente anormales en los días previos y posteriores a anuncios por parte de 85 empresas del NYSE, AMEX y NASDAQ durante los años 1988 a 1993; posteriormente, Busse y Clifton (2002) encuentran que en el año 2000, las noticias públicas no se incorporan de forma instantánea a los precios de los activos, sino que las buenas noticias tardan un minuto, mientras que las negativas se incorporan más lenta y gradualmente en quince minutos; en este mismo sentido, Visaltanachoti y Yang (2010) hallan que en el mercado NYSE

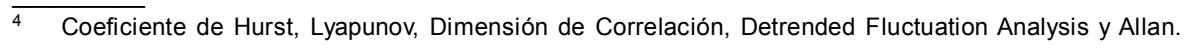


durante el año 2005, las acciones extranjeras toman el doble del tiempo que las acciones locales para reflejar la información pública, dejando en entredicho el mercado de los ADR en este país. Lo anterior llevaría a pensar que desde la década de los ochenta hasta el año 2000, el mercado bursátil de Estados Unidos ha experimentado una mejora en su eficiencia semifuerte, tal como lo plantea Shynkevich (2012).

En la literatura se encuentra que las anomalías de mercado permiten comprobar la hipótesis de eficiencia. Este tipo de estudios fueron ampliamente desarrollados en las décadas de los 70 a los 90 en Norteamérica, es por ello que en la literatura consultada desde 1997 existe un único trabajo sobre anomalías en los Estados Unidos, llevado a cabo por Parhizgari y Nguyen (2008), quienes analizan los activos ADR de origen asiático, europeo, y suramericano en los índices NYSE, AMEX y NASDAQ, desde el año 1982 a 2005, revelando que los ADR en Asia dan beneficios con la estrategia Contrarian mientras que en Europa y Suramérica se dan beneficios con la estrategia Momentum; adicionalmente se verifica efecto tamaño en estos activos.

De acuerdo con lo anterior, se encuentra que en general la literatura muestra un mayor rechazo que aceptación de la hipótesis de mercado eficiente utilizando diferentes metodologías de comprobación como son análisis técnico, modelos de predicción, test de aleatoriedad y caos, análisis de eventos y anomalías de mercado en la región de Norteamérica.

\section{Europa}

A partir de la consulta de investigaciones realizadas desde 1997, solo se encuentran trabajos de Europa para el periodo de 1999 a 2012, lo cual es motivado posiblemente por la unificación monetaria de la región desde el año 2000, que provoca una profunda transformación del sector financiero europeo, tal como lo resalta López (2007) y que a su vez motiva a la comunidad académica a preguntarse sobre la eficiencia de estos mercados bursátiles.

Al igual que en el mercado norteamericano, la hipótesis de eficiencia se ha contrastado en Europa mediante diferentes herramientas y metodologías; entre ellas el análisis técnico en las bolsas de Madrid y Suecia, donde Fernández y González (2000); Straßburg, González, y Alexandrov (2012) y Metghalchi, Chang y Marcucci (2008) encontraron posibilidad de obtener rendimientos extraordinarios para los años 1966 a 1998 y de 1986 a 2004, respectivamente. También los modelos de predicción autorregresivos GARCH, han sido usados en los mercados de Suiza y Alemania por Hess (2003) y Raunig (2006), para hallar predictibilidad de largo plazo desde 1963 a 2005. Otros trabajos que han identificado no eficiencia del mercado europeo mediante análisis de caos y aleatoriedad, están documentados por Opong, 
Mulholland, Fox y Farahmand (1999), quienes rechazan la hipótesis de series independientes e idénticamente distribuidas, y encuentran que el modelo GARCH $(1,1)$ parece explicar los índices del London Financial Times Stock Exchange (FTSE 100-250-350) en el periodo de 1978 a 1997. Igualmente Dicle y Levendis (2011) usan el test de Rachas como un índice de correlación, y encuentran ineficiencia en mercados como el de Grecia, Reino Unido, Alemania y Francia desde 2000 a 2007. Además, Caraiani (2012) rechaza el cumplimiento de la hipótesis de eficiencia en los mercados de República Checa, Hungría y Polonia desde 1993 a 2010, con las pruebas BDS, Rachas, Lyapunov, entre otros. En contraste, otros autores como DePenya y Gil (2007) encuentran evidencia de mejora de la eficiencia desde 1997 en el mercado español con los índices IGBM e IBEX35 mediante pruebas de ratio de varianza y pruebas de autocorrelación; mientras que Khan y Vieito (2012) analizan los mercados de la bolsa de Lisboa-Porto y Euronext, para contrastar la eficiencia débil antes y después de su fusión en 2002, y, mediante pruebas de aleatoriedad, hallan una mejora significativa de eficiencia después de su fusión. Por último, Kasman, Kasman y Torun (2009) obtienen resultados mixtos en cuanto a la eficiencia débil de mercado en los años 90, a partir del uso de modelos de predicción ARFIMA-FIGARCH, que combinan parámetros autorregresivos con comportamientos fractales, y concluyen que se presenta memoria de largo plazo en Estonia y eficiencia en Polonia, Croacia y Bulgaria.

En cuanto a la eficiencia semifuerte, Mazouz y Bowe (2006) concluyen que con el lanzamiento del mercado de futuros (LIFFE) en 2002, las noticias se incorporan más rápidamente a los precios de las acciones subyacentes de Londres (LSE); otro trabajo interesante es llevado a cabo por Lobe y Rieks (2011) quienes realizan un análisis de eventos, para identificar sobrerreacción y anomalías en el mercado de valores de Alemania desde 1988 a 2007; sin embargo, debido a los costos de transacción y el sentimiento del mercado impredecible, estas anomalías difícilmente pueden ser explotadas.

Para el periodo de estudio, el mercado europeo se caracteriza por una mejora de la eficiencia, que según algunos autores, es consecuencia de la consolidación de la comunidad económica y monetaria regional y de la introducción de otros productos financieros como los futuros.

\section{Asia}

Debido a la magnitud del continente asiático, se subdivide por regiones como República Popular China, Oriente y Medio Oriente.

China (incluyendo Hong Kong y Taiwán). A diferencia de los países de Europa del Este y de Rusia, en donde sus economías pasaron de ser 
"planificadas" a "de mercado" de forma relativamente rápida bajo la "teoría de Shock" , China ha llevado a cabo una reforma gradual de su economía en los últimos 35 años ${ }^{6}$, la cual, según Martínez (2001), ha venido ganando adeptos debido a sus excelentes resultados económicos y a su influencia sobre los mercados bursátiles que evidencian una evolución en su eficiencia.

Lo anterior se corrobora en los resultados obtenidos por Groenewold, Kan y Wu (2003), quienes a través de un modelo autorregresivo concluyen que la eficiencia disminuyó cuando los bancos fueron excluidos de la bolsa de valores en 1996 y mejoró cuando fueron readmitidos a principios de 2000. En el 2008, los mismos autores utilizan reglas de análisis técnico desde 1992 a 2001 en las acciones de Shanghái, generando ganancias extraordinarias que desaparecen al incluir los costos de transacción. Años después, Chong et al., (2012) evalúan la eficiencia del mercado chino antes y después de la reforma de las empresas estatales de este país en 2005, usando modelos autorregresivos, y concluyen que esta reforma jugó un papel importante en la mejora de la eficiencia en las dos bolsas de valores. Otro caso concreto del efecto de las reformas es investigado por Fifield y Jetty (2008) y Hung (2009), quienes observan que la liquidez y la eficiencia de las acciones B mejoran después de febrero de 2001, cuando se permitió la inversión de los nacionales en estos activos. No obstante, $\mathrm{Gu}$, Ren, Ni, Chen y Zhou (2010) encuentran memoria de largo plazo al analizar datos intradía de la Bolsa de Shenzhen durante 2003.

Por otra parte, el mercado taiwanés es estudiado por Ammermann y Patterson (2003), quienes evalúan la no linealidad en esta bolsa, y encuentran periodos largos de caminata aleatoria y periodos cortos de alta dependencia lineal y no lineal que no permiten predicciones sistemáticas; así mismo, Cheng y Ying (2009), mediante test normalidad y aleatoriedad, hallan que el efecto solar no es significativo en Taiwán en el periodo de 1971 a 2000; por último, Lu, Shiu y Liu (2012) realizan análisis técnico en este mercado en el periodo de 2002 a 2008 mediante patrones de velas, y encuentran que se pueden obtener ganancias comprando en periodos al alza y sosteniendo en periodos bajistas, pero se pierden después de tomar en cuenta los costos de transacción. En otras palabras, estos tres trabajos no rechazan el cumplimiento de la eficiencia débil en

\footnotetext{
Doctrina Ilamada por Friedman \& Friedman (1980) terapia de shock consiste en una transición rápida de las economías hacia el libre mercado. Una fuerte crítica a esta teoría es presentada por Klein (2007).

6 La cronología de esta reforma está dada por: 1978-1983, incremento de la autonomía administrativa de las empresas estatales; 1984-1988, introducción del «sistema de responsabilidad contractual"; 1989-1992, ralentización de las reformas laborales. Se presiona a las empresas estatales para que creen empleo; 1993-2005, creación de un sistema empresarial moderno, guiado por la política Zhuanda Fangxiao consistente en «amarrar a las grandes y dejar libres a las pequeñas"; Post 2005, Limitación y reversión de la anterior reforma, políticas más igualitarias y populistas, incrementos de subsidios para el sector salud e inversión en el sector público (Fernández L. , 2002).
} 
la bolsa de Taiwán desde 1971 hasta 2008.

En resumen, el progreso económico experimentado por la República Popular China en las últimas décadas, sumado a unas reformas económicas graduales, hacen que este continente haya logrado una evolución positiva en su eficiencia bursátil, la cual ha sido más evidente en el mercado de Taiwán, por ser este uno de los mayores centros económicos $\mathrm{y}$ bursátiles del país.

\section{Oriente}

Corea del Sur Malasia, Filipinas, India, Indonesia, Japón y Singapur. En esta región asiática se han realizado diferentes estudios que coinciden en el rechazo a la eficiencia durante los últimos 25 años. Entre estos autores se destacan Mishra, Sehgal y Bhanumurthy (2011); Jayasinghe y Tsui (2008) y Kang, Cheong y Yoon (2010), quienes analizan el mercado indio, japonés y coreano, respectivamente. Sin embargo, en un estudio más amplio, Sharma y Wongbangpo (2002) detectan eficiencia en el mercado de Filipinas; no obstante rechazan la eficiencia de Malasia, Indonesia, Singapur y Tailandia.

Otros estudios han evaluado la eficiencia de los países del Oriente ${ }^{7}$ (incluido China), entre ellos se destaca el trabajo de Cajueiro y Tabak (2004), quienes encuentran evidencia de memoria a largo plazo principalmente en mercados de Singapur, Shanghái y Shenzhen, sugiriendo que las restricciones de liquidezy de capital podrían jugar un papel importante en la explicación de los resultados de las pruebas de eficiencia del mercado; igualmente, Jiang, Ma y Cai (2007) encuentran correlación serial de largo plazo en las series de esta región; así mismo, Lim y Brooks, Price (2009) mediante un test de bicorrelación demuestran que los límites impuestos en los precios de las acciones y eventos que desestabilizan los mercados de Shenzhen, Shanghái, Corea del Sur y Taiwán son las principales causas de ineficiencia en ellos; además Lao y Singh (2011) evidencian comportamiento de manada desde 1999 a 2009 en India y China, lo que contradice la $\mathrm{EMH}$, en el sentido de que no todos los inversores son racionales y toman decisiones de inversión a partir de los diferentes niveles de información. A pesar de lo expuesto, Hoque, Kim y Pyun (2007) rescatan como eficientes, los mercados de Taiwán y Corea del Sur en el periodo de 1990 a 2004; igualmente Chen, Huang y Lai (2009) hallan eficiencia de mercado mediante análisis técnico para ocho países asiáticos; además Chan, McQueen y Thorley (1998) concluyen que no existen burbujas especulativas racionales antes del año 1994 en seis países asiáticos, lo que contradice los diagnósticos de Del Villar, Murillo y Backal (1998); Ahmed, Barkley

\footnotetext{
Japón, Corea del Sur, Singapur, Taiwán, India, Indonesia, Kuala Lumpur, Malasia, Filipinas, Pakistán, Sri Lanka, Tailandia y Australia.
} 
y Uppal (1999); Porter y Takeuchi (1999); Torrero (2001) y en general, la prensa especializada, quienes detectan burbujas en la valoración de activos financieros e inmobiliarios en el mercado asiático y de Japón a finales de los ochenta y mediados de los noventa. En este mismo sentido, sobre la crisis asiática del 97, Lim, Brooks y Kim (2008) llevan a cabo un interesante estudio donde evalúan la eficiencia de sus mercados en tres subperiodos precrisis, crisis y postcrisis, y llegan a la conclusión de que la crisis afectó negativamente la eficiencia de la mayoría de mercados asiáticos debido a la sobrerreacción de los inversores, provocando un efecto manada ante noticias adversas tanto locales como internacionales que no pudieron ser controladas por las autoridades de estos países. Sin embargo, este autor destaca que después de la crisis la mayoría de economías recuperan su eficiencia de mercado sin argumentar las razones precisas que llevaron a esta mejora.

\section{Oriente Medio $^{8}$}

Bley (2011) utiliza pruebas de aleatoriedad y modelos autorregresivos en media y varianza en cinco mercados del Consejo de Cooperación del Golfo, durante la primera década del 2000, y encuentra que las series no siguen una caminata aleatoria. Por su parte,
Esfahanipour y Mousav (2011) usan programación genética en acciones de la Bolsa de Teherán (Irán), tomando en cuenta los costos de transacción, dividendos y splits, y obtienen ganancias superiores a comprar y mantener para cualquier tendencia del mercado. También, Al-Hajieh, Redhead y Rodgers (2011) contrastan la caminata aleatoria mediante el test de Rachas, y encuentran no aleatoriedad para todos los países islámicos del Medio Oriente excepto Egipto; además se evalúa la anomalía festivo (Mes Ramadán) y hallan retornos positivos durante este mes en el periodo de 1992 a 2007, que son absorbidos por los costos de transacción. Por otra parte, Buguk y Wade (2003) concluyen que Turquía, a diferencia de los demás países, es eficiente en el periodo de 1992 a 1996, y Kasman y Kasman (2008) coinciden en que el mercado turco mejora su eficiencia después de la introducción de los futuros en 2005. Así mismo, Al Janabi, Hatemi-J. y Irandoust (2010) identifican eficiencia semifuerte en el mercado del Consejo de Cooperación del Golfo (GCC) con respecto a las noticias del oro y el petróleo, para el periodo entre 2006 y 2008 .

\section{África}

De los trabajos de Appiah y Menyah (2003); Alagidede (2011) y Gupta y Modise (2013), se desprenden cuatro

$8 \quad$ Turquía, Jordán, Egipto, Kuwait, Arabia Saudí, Emiratos Árabes Unidos, entre otros. Se incluye a Egipto en Oriente Medio y África, debido a que algunos autores lo consideran como parte del uno o el otro. 
grandes conclusiones sobre la eficiencia del continente. En primer lugar, los mercados de Egipto y Marruecos han sido los más eficientes de la región desde el 1990 hasta el 2006; en segundo lugar, se halla una mejora de la eficiencia en los países de Nigeria y Sudáfrica a partir de 1995, motivada posiblemente por el auge de sus exportaciones de materias primas hacia China e India en la última década; en tercer lugar, Kenia pasa de ser un mercado bursátil eficiente del 90 al 95 a no serlo del 95 al 2006, lo cual podría estar relacionado con las crisis económicas y de seguridad que sufrió este país en los noventa, y su posterior recuperación a inicios del 2002; por último, para países como Zimbabue, Mauricio, Túnez, Botsuana, Ghana y Costa de Marfil, no se tiene un registro claro de la trayectoria de su eficiencia de mercado.

\section{Oceanía}

Los estudios eficiencia de mercado de Marshall, Cahan y Cahan (2006) y Bastos y Caiado (2011), contrastan este comportamiento en el mercado neozelandés e identifican una mejora en su eficiencia a partir del año 1992, originada por la transición de un país proteccionista a uno de empresa privada y de mercado, mediante la implementación de reformas en las décadas del 80 y 90 .

Por otro lado, en el mercado australiano existen resultados contradictorios respecto a la eficiencia de mercado, ya que Gaunt (2000) detecta efecto tamaño de empresa en el periodo de 1974 a 1997 , que no puede ser aprovechado debido a la poca liquidez de este mercado; así mismo Ellis y Parbery (2005) evalúan los beneficios producidos por el análisis técnico en los mercados de Australia y obtienen que los retornos no compensan los costos de transacción, por tanto es mejor comprar y mantener durante el periodo de 1980 a 2002; además, Doyle y Chen (2012), usando una prueba de aleatoriedad, no rechazan la hipótesis de que el mercado de Australia sea eficiente en el periodo de 1996 a 2012. Por el contrario, Lee, Lee y Lee (2010) concluyen que el mercado australiano no es eficiente durante el periodo de 1999 a 2007, dado que se presentan dependencias no lineales en las series.

\section{Latinoamérica}

En la base ScienceDirect se encontraron varios trabajos de investigación, entre ellos el de Tabak (2007) y Freitas, De Souza y De Almeida (2009), que evalúan el mercado de Brasil en cuanto a su eficiencia mediante raíces unitarias y modelos de predicción, respectivamente, y revelan que este mercado no es eficiente en su forma débil para el periodo entre 1998 y 2007. Además, los mercados de México y Estados Unidos son evaluados por Mansilla (2001) entre 1996 y 2000, mediante un algoritmo de complejidad física, y hallan aleatoriedad en los retornos de ambos mercados, lo cual es normal teniendo en cuenta la dependencia del mercado mexicano con respecto al de Estados Unidos, tal como 
lo corroboran Atteberry y Swanson (1997) para el periodo de 1985 hasta 1994, y Sarmiento, Duarte y Mascareñas (2012) para el periodo de 2001 a 2010. Por otro lado, Hatgioannides y Mesomeris (2007) encuentran, mediante el análisis técnico, eficiencia en los mercados de Argentina, Brasil, Chile y México, mas no en Indonesia, Filipinas, Taiwán y Tailandia, después de tener en cuenta los costes de transacción para el periodo entre 1988 y 2002.

\section{OTROS ESTUDIOS DE ÁMBITO MUNDIAL}

En la literatura consultada también se encuentran estudios que incluyen al menos dos continentes, los cuales en buena parte se centran en contrastar la eficiencia de países desarrollados con países en vía de desarrollo, tomando como referente de eficiencia a Estados Unidos y Japón, para el Occidente y Oriente, respectivamente.

\section{Estudios a nivel mundial}

Moreno y Olmeda (2007) usan test de aleatoriedad y evalúan la predicción mediante modelos autorregresivos y redes neuronales, sus resultados indican que los modelos no lineales no son mejores que los lineales, y que tanto mercados emergentes como desarrollados no son predecibles, si tienen en cuenta los costos de transacción. Igualmente, Sánchez,
Trinidad y García (2008) evalúan el comportamiento caótico de los índices SyP500, NIKKEI, NASDAQ, IBEX35, en la década de los noventa, y encuentran que no se puede rechazar la hipótesis de que las series sigan una caminata aleatoria. Así mismo, Charles (2010) estudia la predictibilidad de la volatilidad de las acciones en los mercados de Estados Unidos, Europa y Japón, a partir del efecto día de la semana, para verificar que no es posible ganarle al mercado. No obstante, en estudios como el de McKenzie (2001) no se encuentra comportamiento caótico en mercados de Norteamérica, Europa, Australia y Japón, pero identifica dependencias no lineales considerables rechazando la eficiencia de estos mercados; igualmente, Bekiros (2010) analiza la predicción con lógica difusa y redes neuronales en Estados Unidos, Europa y Asia durante 1990 a 2001, y halla posibilidades de ganarle al mercado mediante estos modelos no lineales.

Los anteriores estudios no encuentran diferencias en la eficiencia entre países emergentes y desarrollados; sin embargo, los estudios de Bastos y Caiado (2011) y Eom, Choi, Oh y Jung (2008), hallan relación entre el nivel de eficiencia y de desarrollo de un mercado, y concluyen que los mercados desarrollados son menos predecibles y más eficientes que los emergentes. Por último, Doyle y Chen $(2012)^{9}$, en un estudio más detallado, analizan los mercados bursátiles de 76

9 Este trabajo fue encontrado en la base de datos ScienceDirect, aunque está en proceso de su publicación en European Journal of Operational Research. 
países desde enero del 96 hasta junio de 2012, observan que sus series financieras presentan estructuras, y destacan que los índices de países como Alemania, Austria, Bélgica, España, Francia, Italia, Londres, Países Bajos, Polonia, Suiza, Taiwán, Australia e India no presentan patrones que conduzcan al rechazo ${ }^{10}$ de la hipótesis de aleatoriedad.

\section{Estudios entre dos o más países}

Algunos estudios como los de Ratner y Leal (1999); Chang, Lima y Tabak (2004) y Lim (2007), toman a Estados Unidos y Japón como parámetros de eficiencia para ser comparados con mercados emergentes de Asia y Latinoamérica, revelan que las economías desarrolladas son más eficientes en el periodo comprendido entre 1982 y 2005; así como también lo observa Tse (1998), quien analiza a Japón y Estados Unidos de forma independiente, y verifica eficiencia en ambos mercados. Por el contrario, Skjeltorp (2000) encuentra patrones o tendencias en los rendimientos que persisten a lo largo del tiempo en los mercados de Noruega y Estados Unidos, en el periodo entre 1990 y 1994 . En este mismo sentido, Dionisio, Menezes y Mendes (2004) detectan dependencias lineales y no lineales en Estados Unidos y Europa en el periodo de 1993 a 2002; además Kiliç (2011) usa un modelo FIGARCH y encuentra predictibilidad no lineal en el SyP500 y Turquía. Por su parte, Majumder (2012) contrasta la hipótesis de mercado eficiente mediante el exponente de Hurst en los mercados del BRIC y Estados Unidos para el periodo pre y post de la crisis del 2008; manifiesta que los mercados emergentes, e incluso los desarrollados como el de Estados Unidos, pueden llegar a ser no eficientes debido a las emociones de los inversores.

Finalmente, los mercados del Oriente y Latinoamérica son contrastados mediante modelos autorregresivos por Edwards y Susmel (2001), y verifican fuerte dependencia, especialmente entre los países del Mercosur. Igualmente, estas regiones son estudiadas por Kawakatsu y Morey (1999), quienes al evaluar la premisa de que la eficiencia mejora ante una liberalización del mercado, encuentran que de hecho, los mercados evaluados ya eran eficientes antes de la liberalización real, lo cual lleva a pensar que ante el solo anuncio de que un mercado va a iniciar un proceso de apertura económica, los inversores se anticipan a este hecho, llevando a que el mercado bursátil se haga más eficiente.

\section{Estadísticas}

Al tabular los resultados de las investigaciones (ver Anexo. Estudios por país.) analizadas anteriormente, se observa que:

Los países más testeados en cuanto a su eficiencia del mercado son: Norteamérica

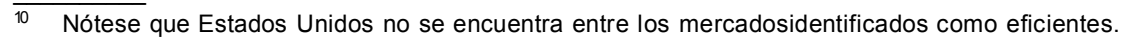


(9,9\%), Taiwán (4,6\%), Corea del Sur (4,1\%), Japón (3,9\%), China $(3,7 \%)$ y Hong Kong (3,0 \%). Por otro lado, los países menos testeados son los pertenecientes a África, seguidos de los países de Medio Oriente y algunos países de Europa Oriental ${ }^{11}$. Para el caso concreto de Latinoamérica, el país más estudiado es Brasil (2,5\%), seguido de México (2,3\%), Argentina (1,8\%), Chile $(1,8 \%)$, Colombia $(1,1 \%)$ y en último lugar Perú $(0,9 \%)$.

La hipótesis de eficiencia de mercado es rechazada en el $60 \%$ de los resultados; no rechazada en un $35 \%$ y se evidencia mejora de eficiencia en un $5 \%$.

Los países con mayor porcentaje de aceptación de eficiencia del mercado en orden descendente son: Francia, Japón, Alemania, Reino Unido, Corea del Sur, Taiwán (China), y por último, Norteamérica.

La mejora de la eficiencia es mayor en los países orientales, principalmente en Corea del Sur, la República Popular China y Tailandia.

\section{CONCLUSIONES}

De la revisión de los antecedentes de investigación en los últimos quince años sobre la hipótesis del mercado eficiente, se encuentra que:
La mayoría de los trabajos consultados (60\%), niega la existencia de la eficiencia del mercado, aunque se debe tener en cuenta que generalmente las investigaciones científicas son dirigidas a refutar una hipótesis con el fin de comprobar su validez, como lo manifiestan Badii y Guillen (2009). Por otra parte, al analizar el $35 \%$ de las investigaciones que no rechazan la hipótesis del mercado eficiente, se encuentra que los países desarrollados ocupan los primeros lugares, lo cual es de esperarse teniendo en cuenta que en estos mercados la información fluye de forma más rápida, a menor coste y además cuentan con un mayor número de participantes que mejoran su liquidez, es decir, son mercados en los cuales se cumplen mejor los supuestos de la hipótesis de eficiencia.

El porcentaje de mercados bursátiles que presentan mejora de su eficiencia en los últimos quince años, es relativamente pequeño (5\%), lo cual puede deberse en parte a que no todos los autores dividen el periodo de estudio en subperiodos que contribuyan a evaluar la evolución de la eficiencia en el tiempo, sin embargo, se encuentra que este comportamiento se observa principalmente en los mercados asiáticos a finales de los noventa, motivado según los autores por:

1. Reformas en las economías que buscan liberalización o menos

11 En su mayoría son países de reciente (o en vía de) vinculación a la Unión Europea. 
restricciones al mercado, que llevan a una mayor liquidez y capitalización, lo que conduce a una mejora de su eficiencia, incluso antes de la implementación de la respectiva reforma, como es verificado por Kawakatsu y Morey (1999). Dos ejemplos se pueden citar: en primera instancia a China, con las diferentes reformas graduales a las empresas estatales y la apertura de su mercado de acciones $\mathrm{B}$ a inversionistas nacionales, y en segundo lugar a Tailandia, con la liberación de su mercado bursátil.

2. Mayor velocidad en el flujo de la información, posibilitado por la Internet, como lo detectado por Lee, Park, Jo, Yang y Moon (2009) en el mercado de Corea del Sur a partir de 1996.

3. Introducción de nuevos productos financieros como las opciones y los futuros, verificada por Mazouz y Bowe (2006) y Kasman y Kasman (2008) en los mercados de Londres (LSE) y Turquía (ISE). Según Salm y Schuppli (2010), estos productos financieros ofrecen información anticipada a bajo coste de los precios spot, lo que contribuye con la mejora de la eficiencia de los respectivos mercados.

4. Periodos postcrisis económicas y bursátiles, que llevan a corregir asimetrías del mercado. Tal como lo comprueban Lim et al. (2008) en los países orientales después de la crisis asiática del 1997.

Existen resultados contradictorios al experimentarse cambios de eficiencia en diferentes periodos para un mismo mercado (principalmente en países desarrollados), hallazgo que es explicado por Majumder (2012), quien argumenta que la eficiencia del mercado no es continua, sino que por el contrario, los mercados bursátiles presentan periodos de eficiencia y no eficiencia. Es así que mercados generalmente aceptados como eficientes pueden presentar periodos de ineficiencia causados por la reacción irracional (optimista o pesimista) de los inversores, quienes en algunos casos motivados por "movimientos del mercado", llevan a cabo estrategias de inversión erróneas de manera colectiva (efecto manada), que ocasionan la formación de asimetrías en los precios de los activos financieros.

El mercado norteamericano es el más testeado debido a que generalmente es usado como parámetro para evaluar la eficiencia relativa de otros mercados. Sin embargo, al consolidar a China continental con Hong Kong y Taiwán en la República Popular China, este país se convierte en el más estudiado en los últimos quince años, lo que reflejaría el interés académico por dicho mercado dado su reciente crecimiento económico. 


\section{Referencias}

Ahmed, E., Barkley, J. \& Uppal, J. (1999). Evidence of nonlinear speculative bubbles in pacificrim stock markets. The quarterly review of economics and finance, 39, 21-36.

Akerlof, G. (1970). The market for "Lemons": Quality uncertainty and the market mechanism. The Quarterly Journal of Economics, 84(3), 488-500.

Al Janabi, M., Hatemi-J, A. \& Irandoust, M. (2010). An empirical investigation of the informational efficiency of the GCC equity markets: Evidence from bootstrap simulation. International Review of Financial Analysis, 19(1), 47-54.

Alagidede, P. (2011). Return behaviour in Africa's emerging equity markets. The Quarterly Review of Economics and Finance, 51(2), 133-140.

Al-Hajieh, H., Redhead, K. \& Rodgers, T. (2011). Investor sentiment and calendar anomaly effects: A case study of the impact of Ramadan on Islamic Middle Eastern markets. Research in International Business and Finance, 25(3), 345-356.

Ammermann, P. \& Patterson, D. (2003). The cross-sectional and cross-temporal universality of nonlinear serial dependencies: Evidence from world stock indices and the Taiwan Stock Exchange. Pacific-Basin Finance Journal, 11(2), 175-195.

Ansari, T., Kumar, M., Shukla, A., Dhar, J. \& Tiwari, R. (2010). Sequential combination of statistics, econometrics and Adaptive Neural-Fuzzy Interface for stock market. Expert Systems with Applications, 37(7), 5116-5125.

Appiah, J. \& Menyah, K. (2003). Return predictability in african stock markets. Review of financial economics, 12(3), 247-270.

Aragonés, J. \& Mascareñas, J. (1994). La eficiencia y el equilibrio en los mercados de capital. Análisis Financiero, 64, 76-89.

Atteberry, W. \& Swanson, P. (1997). Equity market integration: The case of North America. The North American Journal of Economics and Finance, 8(1), 23-37.

Bachelier, L. (1900). Théorie de la spéculation. Annales scientifiques de l'École Normale Supérieure, 17, 21-86.

Badii, M. \& Guillen, A. (2009). Decisiones estadísticas: Bases teóricas: (Statistical Decision Making: Theoretical Basis). International Journal of Good Conscience, 5, 185-207.

Bastos, J. \& Caiado, J. (2011). Recurrence quantification analysis of global stock markets. Physica A: Statistical Mechanics and its Applications, 390(7), 1315-1325.

Bekiros, S. (2010). Fuzzy adaptive decision-making for boundedly rational traders in speculative stock markets. European Journal of Operational Research, 202, 285-293. 
Bley, J. (2011). Are GCC stock markets predictable? Emerging Markets Review, 12(3), 217-237.

Brock, W., Lakonishok, J. \& LeBaron, B. (1992). Simple technical trading rules and the stochastic: properties of stock returns. Journal of Finance, 47(5), 1731-1764.

Buguk, C. \& Wade, B. (2003). Testing weak-form market efficiency: Evidence from the Istanbul Stock Exchange. International Review of Financial Analysis, 12(5), 579-590.

Busse, J. \& Clifton, T. (2002). Market efficiency in real time. Journal of Financial Economics, 65(3), 415-437.

Cajueiro, D. \& Tabak, B. (2004). Evidence of long range dependence in Asian equity markets: the role of liquidity and market restrictions. Physica A: Statistical Mechanics and its Applications, 342(3-4), 656-664.

Caraiani, P. (2012). Nonlinear dynamics in CEE stock markets indices. Economics Letters, $114(3), 329-331$.

Cardano, G. (1953). The Book on Games of Chance (Liber de Ludo Aleae). (S. H. Gould, Trad.) Nueva York: Holt, Rinehart and Winston.

Coakley, J. \& Fuertes, A. (2006). Valuation ratios and price deviations from fundamentals. Journal of Banking and Finance, 30(8), 2325-2346.

Couillard, M. \& Davison, M. (2005). A comment on measuring the Hurst exponent of financial time series. Physica A: Statistical Mechanics and its Applications, 348, 404-418.

Cowles, A. (1933). Can stock market forecasters forecast? Econometrica, 309-324.

Chan, K., McQueen, G. \& Thorley, S. (1998). Are there rational speculative bubbles in Asian stock markets? Pacific-Basin Finance Journal, 6(1-2), 125-151.

Chang, E., Lima, E. \& Tabak, B. (2004). Testing for predictability in emerging equity markets. Emerging Markets Review, 5(3), 295-316.

Charles, A. (2010). The day-of-the-week effects on the volatility: The role of the asymmetry. European Journal of Operational Research, 202(1), 143-152.

Chen, C. W., Gerlach, R. \& Liu, F.-C. (2011). Detection of structural breaks in a time-varying heteroskedastic regression model. Journal of Statistical Planning and Inference, 141(11), 3367-3381.

Chen, C., Huang, C. \& Lai, H. (2009). The impact of data snooping on the testing of technical analysis: An empirical study of Asian stock markets. Journal of Asian Economics, 20(5), 580-591.

Cheng, H. \& Ying, K. (2009). Testing the significance of solar term effect in the Taiwan stock market. Expert Systems with Applications, 36(3, Part 2), 6140-6144. 
Chong, T., Lam, T. \& Yan, I. (2012). Is the Chinese stock market really inefficient? China Economic Review, 23(1), 122-137.

Day, T. \& Wang, P. (2002). Dividends, nonsynchronous prices, and the returns from trading the DJIA. Journal of empirical finance, 9(4), 431-454.

Del Villar, R., Murillo, J. \& Backal, D. (1998). La crisis financiera en Asia: orígenes y evolución en 1997 y 1998. Dirección General de Investigación Económica. Banco de México, 42.

DePenya, F. J. \& Gil, L. (2007). Serial correlation in the Spanish stock market. Global Finance Journal, 18, 84-103.

Dicle, M. \& Levendis, J. (2011). Greek market efficiency and its international integration. Journal of International Financial Markets, Institutions and Money, 21(2), 229-246.

Dionisio, A., Menezes, R. \& Mendes, D. (2004). Mutual information: a measure of dependency for nonlinear time series. Physica A: Statistical Mechanics and its Applications, 344(12), 326-329.

Doyle, J. \& Chen, C. (2012). Patterns in stock market movements tested as random number generators. European Journal of Operational Research, 227(1), 122-132.

Easley, D., Kiefer, N. \& O’Hara, M. (1997). The information content of the trading process. Journal of Empirical Finance, 4(2-3), 159-186.

Edwards, S. \& Susmel, R. (2001). Volatility dependence and contagion in emerging equity markets. Journal of Development Economics, 66(2), 505-532.

Ellis, C. \& Parbery, S. (2005). Is smarter better? A comparison of adaptive, and simple moving average trading strategies. Research in International Business and Finance, 19(3), 399411.

Eom, C., Choi, S., Oh, G. \& Jung, W. (2008). Hurst exponent and prediction based on weak-form efficient market hypothesis of stock markets. Physica A: Statistical Mechanics and its Applications, 387(18), 4630-4636.

Esfahanipour, A. \& Mousavi, S. (2011). A genetic programming model to generate risk-adjusted technical trading rules in stock markets. Expert Systems with Applications, 38(7), 84388445 .

Fama, E. (1965). The behavior of stock-market prices. Journal of business, 38(1), 34-105.

Fama, E. (1970). Efficient capital markets: A review of theory and empirical work. Journal of Finance, 25, 383-417.

Fama, E. (1991). Efficient capital markets II. The journal of finance, 46(5), 1575-1617. 
Fernández, F. \& González, C. (2000). Optimización de reglas técnicas en el IGBM usando algoritmos genéticos. Comunicaciones XIV Reunión: Anales de Economía Aplicada. Oviedo.

Fernández, L. (2002). Reformas de las empresas estatales y politica de reempleo en China. Revista ICE, 797, 101-117.

Ferreira, E. \& Brooks, L. (1999). Evidence on equity private placements and going-out-ofbusiness information release. Journal of economics and business, 51(5), 377-394.

Fifield, S. \& Jetty, J. (2008). Further evidence on the efficiency of the Chinese stock markets: A note. Research in International Business and Finance, 22(3), 351-361.

Freitas, F., De Souza, A. \& De Almeida, A. (2009). Prediction-based portfolio optimization model using neural networks. Neurocomputing, 72(10-12), 2155-2170.

Friedman, M. \& Friedman, R. (1980). Free to choose: A personal statement. (C. R. Pujol, Trad.) Nueva York: Ediciones Orbis S.A.

Gaunt, C. (2000). Overreaction in the Australian equity market: 1974-1997. Pacific-Basin Finance Journal, 8(3-4), 375-398.

Gençay, R. (1998). The predictability of security returns with simple technical trading rules. Journal of Empirical Finance, 5(4), 347-359.

Groenewold, N., Kan, S. \& Wu, Y. (2003). The efficiency of the Chinese stock market and the role of the banks. Journal of Asian Economics, 14(4), 593-609.

Grossman, S., y Stiglitz, J. (1980). On the Impossibility of Informationally Efficient Markets. 70(3), 393-408.

Gu, G., Ren, F., Ni, X., Chen, W. \& Zhou, W. (2010). Empirical regularities of opening call auction in Chinese stock market. Physica A: Statistical Mechanics and its Applications, $389(2), 278-286$.

Gupta, R. \& Modise, M. (2013). Macroeconomic variables and south african stock return predictability. Economic Modelling, 30, 612-622.

Hatgioannides, J. \& Mesomeris, S. (2007). On the returns generating process and the profitability of trading rules in emerging capital markets. Journal of International Money and Finance, 26(6), 948-973.

Hess, M. (2003). What drives Markov regime-switching behavior of stock markets? The Swiss case. International Review of Financial Analysis, 12(5), 527-543.

Hoque, H., Kim, J. \& Pyun, C. (2007). A comparison of variance ratio tests of random walk: A case of Asian emerging stock markets. International Review of Economics y Finance, $16(4), 488-502$. 
Hung, J. (2009). Deregulation and liberalization of the Chinese stock market and the improvement of market efficiency. The Quarterly Review of Economics and Finance, 49(3), 843-857.

Jayasinghe, P. \& Tsui, A. (2008). Exchange rate exposure of sectoral returns and volatilities: Evidence from Japanese industrial sectors. Japan and the World Economy, 20(4), 639660.

Jiang, J., Ma, K. \& Cai, X. (2007). Non-linear characteristics and long-range correlations in Asian stock markets. Physica A: Statistical Mechanics and its Applications, 378(2), 399-407.

Kaminsky, G. \& Schmukler, S. (1999). What triggers market jitters? A chronicle of the Asian crisis. Journal of International Money and Finance, 18, 537"560.

Kang, S., Cheong, C. \& Yoon, S. (2010). Long memory volatility in Chinese stock markets. Physica A: Statistical Mechanics and its Applications, 389(7), 1425-1433.

Kasman, A. \& Kasman, S. (2008). The impact of futures trading on volatility of the underlying asset in the Turkish stock market. Physica A: Statistical Mechanics and its Applications, 387(12), 2837-2845.

Kasman, A., Kasman, S. \& Torun, E. (2009). Dual long memory property in returns and volatility: Evidence from the CEE countries' stock markets. Emerging Markets Review, 10(2), 122139.

Kawakatsu, H. \& Morey, M. (1999). Financial liberalization and stock market efficiency: an empirical examination of nine emerging market countries. Journal of Multinational Financial Management, 9(3-4), 353-371.

Kendall, M. (1953). The analysis of economic time-series-part I: prices. Journal of the Royal Statistical Society. Series A (General), 116, 11-25.

Khan, W. \& Vieito, J. (2012). Stock exchange mergers and weak form of market efficiency: The case of Euronext Lisbon. International Review of Economics y Finance, 22(1), 173-189.

Kiliç, R. (2011). Long memory and nonlinearity in conditional variances: A smooth transition FIGARCH model. Journal of Empirical Finance, 18(2), 368-378.

Kim, J., Shamsuddin, A. \& Lim, K. (2011). Stock return predictability and the adaptive markets hypothesis: Evidence from century-long U.S. data. Journal of Empirical Finance, 18(5), 868-879.

Klein, N. (2007). La doctrina del shock: El auge del capitalismo del desastre. Knopf, Canada: Editorial Paidos. 


\section{APUNTES DEL CENES N ${ }^{\circ} .57$}

Vol. 33, Enero - Junio de 2014

Kohers, T., Pandey, V. \& Kohers, G. (1997). Using nonlinear dynamics to test for market efficiency among the major U.S. stock exchanges. The Quarterly Review of Economics and Finance, 37(2), 523-545.

Lao, P. \& Singh, H. (2011). Herding behaviour in the Chinese and Indian stock markets. Journal of Asian Economics, 22(6), 495-506.

Lee, C., Lee, J. \& Lee, C. (2010). Stock prices and the efficient market hypothesis: Evidence from a panel stationary test with structural breaks. Japan and the World Economy, 22(1), 49-58.

Lee, J., Park, J., Jo, H., Yang, J. \& Moon, H. (2009). Minimum entropy density method for the time series analysis. Physica A: Statistical Mechanics and its Applications, 388(2-3), $137-144$.

Lim, K. (2007). Ranking market efficiency for stock markets: A nonlinear perspective. Physica A: Statistical Mechanics and its Applications, 376, 445-454.

Lim, K. \& Brooks, R. (2009). Price limits and stock market efficiency: Evidence from rolling bicorrelation test statistic. Chaos, Solitons y Fractals, 40(3), 1271-1276.

Lim, K., Brooks, R. \& Kim, J. (2008). Financial crisis and stock market efficiency: Empirical evidence from Asian countries. International Review of Financial Analysis, 17(3), 571591.

Liu, S. (2007). International cross-listing and stock pricing efficiency: An empirical study. Emerging Markets Review, 8(4), 251-263.

Lobe, S. \& Rieks, J. (2011). Short-term market overreaction on the Frankfurt stock exchange. The Quarterly Review of Economics and Finance, 51(2), 113-123.

López, I. (2007). El proceso de integración de los mercados financieros en Europa. Escuela de Administración de Negocios, 59, 87-97.

Lu, T., Shiu, Y. \& Liu, T. (2012). Profitable candlestick trading strategies-The evidence from a new perspective. Review of Financial Economics, 21(2), 63-68.

Ludlow, J. (1997). Modelos, pronósticos y volatilidad de las series de tiemo generadas en la bolsa mexicana de valores. Azcapotzalco: Universidad Autónoma Metropolitana Azcapotzalco.

Majumder, D. (2012). When the market becomes inefficient: Comparing BRIC markets with markets in the USA. International Review of Financial Analysis, 24, 84-92.

Malkiel, B. (1992). Efûcient market hypothesis. En M. M. P. Newman (Ed.), New Palgrave Dictionary of Money and Finance. Londres: Macmillan. 
Mandelbrot, B. (1963). New methods in statistical economics. Journal of Political Economy, $71,421-440$.

Mansilla, R. (2001). Algorithmic complexity of real financial markets. Physica A: Statistical Mechanics and its Applications, 301(1-4), 483-492.

Marshall, B., Cahan, J., y Cahan, R. (2006). Is the CRISMA technical trading system profitable? Global Finance Journal, 17(2), 271-281.

Marshall, B., Young, M. \& Rose, L. (2006). Candlestick technical trading strategies: Can they create value for investors? Journal of Banking y Finance, 30(8), 2303-2323.

Martínez, M. (Junio de 2001). Privatizaciones y Reforma del Sector Público en China. Recuperado el Diciembre de 2012, de ICEX: España Exportación e Inversiones: http:// www.icex.es/servicios/documentacion/documentoselaborados/icex/pdfs/ privatizaciones\%20reformas\%20sector\%20publico\%20china.pdf

Mazouz, K. \& Bowe, M. (2006). The volatility effect of futures trading: Evidence from LSE traded stocks listed as individual equity futures contracts on LIFFE. International Review of Financial Analysis, 15(1), 1-20.

McKenzie, M. (2001). Chaotic behavior in national stock market indices: New evidence from the close returns test. Global Finance Journal, 12(1), 35-53.

Metghalchi, M., Chang, Y. \& Marcucci, J. (2008). Is the Swedish stock market efficient? Evidence from some simple trading rules. International Review of Financial Analysis, 17(3), 475490.

Mishra, R., Sehgal, S. \& Bhanumurthy, N. (2011). A search for long-range dependence and chaotic structure in Indian stock market. Review of Financial Economics, 20(2), 96-104.

Moreno, D. \& Olmeda, I. (2007). Is the predictability of emerging and developed stock markets really exploitable? European Journal of Operational Research, 182(1), 436-454.

Mulligan, R. \& Lombardo, G. (2004). Maritime businesses: volatile stock prices and market valuation inefficiencies. The Quarterly Review of Economics and Finance, 44(2), 321336.

Opong, K., Mulholland, G., Fox, A. \& Farahmand, K. (1999). The behaviour of some UK equity indices: An application of Hurst and BDS tests. Journal of Empirical Finance, 6(3), 267282.

Osborne, M. (1959). Brownian motion in the stock market. Operations Research, 7(2), 145733.

Parhizgari, A. \& Nguyen, D. (2008). ADRs under momentum and contrarian strategies. Global Finance Journal, 19(2), 102-122. 
Peters, E. (1994). Fractal market analysis: Applying chaos theory to investment and economics. Nueva York: Wiley Finance Editions.

Porter, M. \& Takeuchi, H. (1999). Fixing what really ails japan. Foreign Affairs, 3, 66-81.

Potvin, J., Soriano, P. \& Vallée, M. (2004). Generating trading rules on the stock markets with genetic programming. Computers and Operations Research, 31(7), 1033-1047.

Ratner, M. \& Leal, R. (1999). Tests of technical trading strategies in the emerging equity markets of Latin America and Asia. Journal of banking y finance, 23(12), 1887-1905.

Raunig, B. (2006). The longer-horizon predictability of German stock market volatility. International Journal of Forecasting, 22(2), 363-372.

Roberts, H. (1959). Stock market "patterns" and financial analysis: Methodological suggestions. The Journal of Finance, 14, 1-10.

Roberts, H. (1967). Statistical versus clinical prediction of the stock market. Chicago: Unpublished manuscript, University of Chicago.

Salm, C. \& Schuppli, M. (2010). Positive feedback trading in stock index futures: International evidence. International Review of Financial Analysis, 19(5), 313-322.

Samuelson, P. (1965). Proof that properly anticipated prices fluctuate randomly. Industrial Management Review, 6(2), 41-49.

Sánchez, M., Trinidad, J. \& García, J. (2008). Some comments on Hurst exponent and the long memory processes on capital markets. Physica A: Statistical Mechanics and its Applications, 387(22), 5543-5551.

Sarmiento, P., Duarte, J. \& Mascareñas, J. (2012). Análisis de causalidad entre mercados bursátiles latinoamericanos Y el Standard y Poor's. 1er Congreso Global de Contabilidad y Finanzas. Bogotá: Universidad Nacional.

Serletis, A. \& Shintani, M. (2003). No evidence of chaos but some evidence of dependence in the US stock market. Chaos, Solitons y Fractals, 17(2-3), 449-454.

Sharma, S. \& Wongbangpo, P. (2002). Long-term trends and cycles in ASEAN stock markets. Review of Financial Economics, 11(4), 299-315.

Shleifer, A. (2003). Are financial markets efficient? En A. Shleifer, Inefficient Markets:An Introduction to Behavioral Finance. Oxford: Oxford University Press.

Shynkevich, A. (2012). Short-term predictability of equity returns along two style dimensions. Journal of Empirical Finance, 19(5), 675-685. 
Skjeltorp, J. (2000). Scaling in the Norwegian stock market. Physica A: Statistical Mechanics and its Applications, 283(3-4), 486-528.

Spence, M. (1973). Job Market Signaling. The quarterly journal of Economics, 87(3), 355-374.

Stiglitz, J. (2010). Freefall: America, free markets, and the sinking of the world economy. Nueva York: W. W. Norton.

Stiglitz, J. \& Rothschild, M. (1976). Equilibrium in competitive insurance markets: an essay on the economics of imperfect information. The Quarterly Journal of Economics, 90(4), 629649 .

Straßburg, J., González, C. \& Alexandrov, V. (2012). Parallel genetic algorithms for stock market trading rules. Procedia Computer Science, 9, 1306-1313.

Tabak, B. (2007). Testing for unit root bilinearity in the Brazilian stock market. Physica A: Statistical Mechanics and its Applications, 385(1), 261-269.

Torrero, A. (2001). El final de la burbuja especulativa y la crisis económica de Japón. Economiaz, 48(3), $92-127$.

Tse, Y. (1998). International transmission of information: evidence from the Euroyen and Eurodollar futures markets. Journal of International Money and Finance, 17(6), 909929.

Uribe, J. \& Ulloa, I. (2011). Revisando la hipótesis de los mercados eficientes: Nuevos datos, nueva crisis, nuevas estimaciones. Banco de la República. 204. Bogotá: Seminario de economía.

Vayanos, D. \& Woolley, P. (2013). An institutional theory of momentum and reserval. Review of Financial Studies, 26(5), 1087-1145.

Visaltanachoti, N. \& Yang, T. (2010). Speed of convergence to market efficiency for NYSElisted foreign stocks. Journal of Banking y Finance, 34(3), 594-605.

Wu, P., Huang, C. \& Chiu, C. (2011). Effects of structural changes on the risk characteristics of REIT returns. International review of economics y finance, 20(4), 645-653. 
ANEXO. Estudios por país.

\begin{tabular}{|c|c|c|c|c|c|c|c|}
\hline \multirow{2}{*}{\begin{tabular}{|l|} 
País \\
Alemania
\end{tabular}} & \multicolumn{2}{|c|}{$\begin{array}{c}\text { No rechaza } \\
\text { EMH }\end{array}$} & \multicolumn{2}{|c|}{$\begin{array}{c}\text { Rechaza } \\
\text { EMH }\end{array}$} & \multicolumn{2}{|c|}{$\begin{array}{c}\text { Mejora de } \\
\text { la eficiencia }\end{array}$} & \multirow{2}{*}{$\begin{array}{c}\begin{array}{c}\text { Total estu- } \\
\text { dios por país }\end{array} \\
11\end{array}$} \\
\hline & 5 & $45 \%$ & 6 & $55 \%$ & 0 & $0 \%$ & \\
\hline Arabia Saudí & 0 & $0 \%$ & 4 & $100 \%$ & 0 & $0 \%$ & 4 \\
\hline Argentina & 4 & $40 \%$ & 5 & $50 \%$ & 1 & $10 \%$ & 10 \\
\hline Australia & 4 & $40 \%$ & 6 & $60 \%$ & 0 & $0 \%$ & 10 \\
\hline Austria & 3 & $60 \%$ & 2 & $40 \%$ & 0 & $0 \%$ & 5 \\
\hline Bangladesh & 0 & $0 \%$ & 2 & $100 \%$ & 0 & $0 \%$ & 2 \\
\hline Bahréin & 1 & $50 \%$ & 1 & $50 \%$ & 0 & $0 \%$ & 2 \\
\hline Bélgica & 3 & $60 \%$ & 2 & $40 \%$ & 0 & $0 \%$ & 5 \\
\hline Botsuana & 0 & $0 \%$ & 2 & $100 \%$ & 0 & $0 \%$ & 2 \\
\hline Brasil & 4 & $29 \%$ & 9 & $64 \%$ & 1 & $7 \%$ & 14 \\
\hline Bulgaria & 1 & $100 \%$ & 0 & $0 \%$ & 0 & $0 \%$ & 1 \\
\hline Chile & 4 & $40 \%$ & 5 & $50 \%$ & 1 & $10 \%$ & 10 \\
\hline China Continental $^{12}$ & 4 & $19 \%$ & 14 & $67 \%$ & 3 & $14 \%$ & 21 \\
\hline Colombia & 2 & $33 \%$ & 3 & $50 \%$ & 1 & $17 \%$ & 6 \\
\hline Corea del Sur & 9 & $39 \%$ & 10 & $43 \%$ & 4 & $17 \%$ & 23 \\
\hline Costa de Marfil & 0 & $0 \%$ & 1 & $100 \%$ & 0 & $0 \%$ & 1 \\
\hline Croacia & 1 & $50 \%$ & 1 & $50 \%$ & 0 & $0 \%$ & 2 \\
\hline Dinamarca & 2 & $50 \%$ & 2 & $50 \%$ & 0 & $0 \%$ & 4 \\
\hline Egipto & 3 & $60 \%$ & 2 & $40 \%$ & 0 & $0 \%$ & 5 \\
\hline Emiratos & 1 & $50 \%$ & 1 & $50 \%$ & 0 & $0 \%$ & 2 \\
\hline Eslovaquia & 0 & $0 \%$ & 2 & $100 \%$ & 0 & $0 \%$ & 2 \\
\hline Eslovenia & 1 & $25 \%$ & 3 & $75 \%$ & 0 & $0 \%$ & 4 \\
\hline España & 4 & $44 \%$ & 4 & $44 \%$ & 1 & $11 \%$ & 9 \\
\hline Estonia & 0 & $0 \%$ & 3 & $100 \%$ & 0 & $0 \%$ & 3 \\
\hline Finlandia & 2 & $67 \%$ & 1 & $33 \%$ & 0 & $0 \%$ & 3 \\
\hline Filipinas & 3 & $19 \%$ & 12 & $75 \%$ & 1 & $6 \%$ & 16 \\
\hline Francia & 7 & $58 \%$ & 5 & $42 \%$ & 0 & $0 \%$ & 12 \\
\hline Ghana & 0 & $0 \%$ & 1 & $100 \%$ & 0 & $0 \%$ & 1 \\
\hline Grecia & 2 & $29 \%$ & 5 & $71 \%$ & 0 & $0 \%$ & 7 \\
\hline Holanda & 3 & $60 \%$ & 2 & $40 \%$ & 0 & $0 \%$ & 5 \\
\hline Hong Kong & 4 & $24 \%$ & 12 & $71 \%$ & 1 & $6 \%$ & 17 \\
\hline Hungría & 2 & $33 \%$ & 4 & $67 \%$ & 0 & $0 \%$ & 6 \\
\hline India & 4 & $29 \%$ & 9 & $64 \%$ & 1 & $7 \%$ & 14 \\
\hline Indonesia & 3 & $20 \%$ & 11 & $73 \%$ & 1 & $7 \%$ & 15 \\
\hline Irán & 0 & $0 \%$ & 1 & $100 \%$ & 0 & $0 \%$ & 1 \\
\hline Irlanda & 1 & $50 \%$ & 1 & $50 \%$ & 0 & $0 \%$ & 2 \\
\hline Islandia & 0 & $0 \%$ & 1 & $100 \%$ & 0 & $0 \%$ & 1 \\
\hline Israel & 2 & $100 \%$ & 0 & $0 \%$ & 0 & $0 \%$ & 2 \\
\hline Italia & 4 & $67 \%$ & 2 & $33 \%$ & 0 & $0 \%$ & 6 \\
\hline Jamaica & 0 & $0 \%$ & 1 & $100 \%$ & 0 & $0 \%$ & 1 \\
\hline Japón & 11 & $50 \%$ & 11 & $50 \%$ & 0 & $0 \%$ & 22 \\
\hline
\end{tabular}

Fuente: elaboración del autor.

\footnotetext{
$12 \quad$ Incluye las bolsas de Shenzhen, Shanghái y en menor proporción Pekín.
} 


\begin{tabular}{|c|c|c|c|c|c|c|c|}
\hline País & & $\begin{array}{l}\text { echaza } \\
\text { MH }\end{array}$ & & $\begin{array}{l}\text { haza } \\
\text { MH }\end{array}$ & & $\begin{array}{l}\text { ra de } \\
\text { iencia }\end{array}$ & $\begin{array}{l}\text { Total estu- } \\
\text { dios por país }\end{array}$ \\
\hline Jordán & 1 & $50 \%$ & 1 & $50 \%$ & 0 & $0 \%$ & 2 \\
\hline Kenia & 1 & $25 \%$ & 3 & $75 \%$ & 0 & $0 \%$ & 4 \\
\hline Kuala Lumpur & 2 & $100 \%$ & 0 & $0 \%$ & 0 & $0 \%$ & 2 \\
\hline Kuwait & 1 & $33 \%$ & 2 & $67 \%$ & 0 & $0 \%$ & 3 \\
\hline Luxemburgo & 1 & $33 \%$ & 2 & $67 \%$ & 0 & $0 \%$ & 3 \\
\hline Malasia & 3 & $19 \%$ & 12 & $75 \%$ & 1 & $6 \%$ & 16 \\
\hline Marruecos & 3 & $60 \%$ & 2 & $40 \%$ & 0 & $0 \%$ & 5 \\
\hline Mauricio & 1 & $50 \%$ & 1 & $50 \%$ & 0 & $0 \%$ & 2 \\
\hline México & 4 & $31 \%$ & 8 & $62 \%$ & 1 & $8 \%$ & 13 \\
\hline Nigeria & 1 & $25 \%$ & 3 & $75 \%$ & 0 & $0 \%$ & 4 \\
\hline Norteamérica $^{13}$ & 19 & $34 \%$ & 36 & $64 \%$ & 1 & $2 \%$ & 56 \\
\hline Noruega & 1 & $25 \%$ & 3 & $75 \%$ & 0 & $0 \%$ & 4 \\
\hline Nueva Zelanda & 2 & $33 \%$ & 3 & $50 \%$ & 1 & $17 \%$ & 6 \\
\hline Omán & 1 & $33 \%$ & 2 & $67 \%$ & 0 & $0 \%$ & 3 \\
\hline Pakistán & 2 & $40 \%$ & 3 & $60 \%$ & 0 & $0 \%$ & 5 \\
\hline Perú & 2 & $40 \%$ & 3 & $60 \%$ & 0 & $0 \%$ & 5 \\
\hline Polonia & 4 & $57 \%$ & 3 & $43 \%$ & 0 & $0 \%$ & 7 \\
\hline Portugal & 2 & $25 \%$ & 5 & $63 \%$ & 1 & $13 \%$ & 8 \\
\hline Qatar & 1 & $50 \%$ & 1 & $50 \%$ & 0 & $0 \%$ & 2 \\
\hline República Checa & 2 & $40 \%$ & 3 & $60 \%$ & 0 & $0 \%$ & 5 \\
\hline Rusia & 2 & $40 \%$ & 3 & $60 \%$ & 0 & $0 \%$ & 5 \\
\hline Singapur & 4 & $25 \%$ & 11 & $69 \%$ & 1 & $6 \%$ & 16 \\
\hline Sri Lanka & 2 & $33 \%$ & 4 & $67 \%$ & 0 & $0 \%$ & 6 \\
\hline Suazilandia & 0 & $0 \%$ & 1 & $100 \%$ & 0 & $0 \%$ & 1 \\
\hline Sudáfrica & 3 & $43 \%$ & 4 & $57 \%$ & 0 & $0 \%$ & 7 \\
\hline Suecia & 2 & $40 \%$ & 3 & $60 \%$ & 0 & $0 \%$ & 5 \\
\hline Suiza & 4 & $50 \%$ & 4 & $50 \%$ & 0 & $0 \%$ & 8 \\
\hline Tailandia & 3 & $20 \%$ & 10 & $67 \%$ & 2 & $13 \%$ & 15 \\
\hline Taiwán & 9 & $35 \%$ & 16 & $62 \%$ & 1 & $4 \%$ & 26 \\
\hline Trinidad y Tobago & 0 & $0 \%$ & 1 & $100 \%$ & 0 & $0 \%$ & 1 \\
\hline Túnez & 0 & $0 \%$ & 2 & $100 \%$ & 0 & $0 \%$ & 2 \\
\hline Turquía & 2 & $33 \%$ & 3 & $50 \%$ & 1 & $17 \%$ & 6 \\
\hline Ucrania & 0 & $0 \%$ & 1 & $100 \%$ & 0 & $0 \%$ & 1 \\
\hline Reino Unido & 6 & $40 \%$ & 8 & $53 \%$ & 1 & $7 \%$ & 15 \\
\hline Venezuela & 2 & $67 \%$ & 0 & $0 \%$ & 1 & $33 \%$ & 3 \\
\hline Zambia & 0 & $0 \%$ & 1 & $100 \%$ & 0 & $0 \%$ & 1 \\
\hline Zimbabue & 1 & $50 \%$ & 1 & $50 \%$ & 0 & $0 \%$ & 2 \\
\hline
\end{tabular}

Fuente: elaboración del autor.

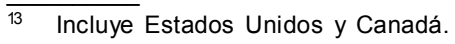

